\title{
Effects of flickering light on the attraction of nocturnal insects
}

The Society of Light and Lighting

\author{
A Barroso $\mathrm{PhD}^{\mathrm{a}}$, I Haifig $\mathrm{PhD}^{\mathrm{b}}$, V Janei $\mathrm{MSc}^{\mathrm{b}}$, I da Silva ${ }^{\mathrm{b}}, \mathbf{C}$ Dietrich $\mathrm{PhD}^{\mathrm{b}}$ and \\ AM Costa-Leonardo $\mathrm{PhD}^{\mathrm{b}}$

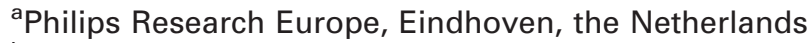 \\ bInstituto de Biociências, Universidade Estadual Paulista, Rio Claro, Brazil
}

Received 25 April 2015; Revised 30 June 2015; Accepted 29 July 2015

\begin{abstract}
We tested the hypothesis that the phototatic response of nocturnal insects is influenced by the flickering of light sources by comparing the numbers of insects captured in traps illuminated with flickering and non-flickering light. Four flicker profiles produced by a square pulse wave with different combinations of frequency and duty cycle were investigated. Overall, fewer insects were captured in traps illuminated with a flickering light source, independent of the flicker setting used. Furthermore, the difference observed was statistically significant for specific combinations of flickering conditions and insect orders, thus suggesting that flickering reduces the number of nocturnal insects attracted to light sources.
\end{abstract}

\section{Introduction}

Insects perform vital functions in ecosystems, e.g. flower pollination, decomposition of organic matter and nutrient recycling. However, several species of insects are pests, inflicting damage to property and transmitting diseases such as Malaria, Dengue, Yellow Fever, Chagas and Leshmaniasis.

Some insects display positive phototaxis, i.e. a tendency to move towards a stimulus of light. This behaviour explains why insects are often seen hovering around lit artificial light sources. The elicited movement of insects towards artificial light sources represents a hazard to humans by promoting the migration of harmful species to inhabited areas, ${ }^{1}$ as in the case of triatomines, carriers of Chagas disease that infest houses primarily close to public street lighting. ${ }^{2}$ Insect migration to artificially lit areas also threatens the biodiversity of surrounding ecosystems as

Address for correspondence: Andre Barroso, Philips Research Europe, High Tech Campus 34, 5656AE Eindhoven, the Netherlands.

E-mail: andre.barroso@philips.com indicated by a recent study in a German city of 240,000 inhabitants, which estimated that around 360 million insects die per season after being attracted to street lamps. ${ }^{3}$ Generalpurpose artificial lighting sources that elicit little or no insect phototaxis would thus contribute to preserving both human wellbeing and ecosystems.

Current commercial lamps designed to minimise insect attraction emit a non-white light, which restricts their use, e.g. for porch or garden illumination. Such lamps leverage the knowledge that insect phototaxis depends on the specific spectrum of light to reduce energy in wavelengths that are most attractive to insects. ${ }^{4-10}$ As some of these wavelengths are visible to humans, their removal impoverishes the colour rendering of the light source. A general-purpose lamp that minimises insect attraction thus remains elusive.

Light emitting diode (LED) technology offers almost full control of the spectrum and polarisation of the light emitted and permits a fast output response to variations in the driving current. Such flexibility represents a unique opportunity for the investigation of 
artificial light properties that affect insect phototaxis. In particular, the influence of temporal artefacts on the movement of flying insects is a promising area of research as flicker may introduce stroboscopic effects that impair flight control strategies. ${ }^{11}$

The photoreceptors of fast-moving insects such as honeybees must sample the environment at high frequencies in order to enable collision-free manoeuvres. The temporal resolution at which an animal samples its environment can be assessed by the threshold frequency at which it ceases to perceive a flickering light source as a series of flashes and instead perceives a continuous stream of light. This threshold, known as the critical fusion frequency (CFF), has an average value of approximately $60 \mathrm{~Hz}$ for humans and $240 \mathrm{~Hz}$ for honeybees. ${ }^{12}$ A recent meta-study summarising a large quantity of CFF values across a broad range of taxa indicates that insects have by far the highest CFF mean values, although a distinct difference between nocturnal (mean $70 \mathrm{~Hz}$ ) and diurnal species $(\text { mean } 201 \mathrm{~Hz})^{12}$ was observed. The high CFF values of insects suggest that many species perceive flicker that humans do not, which opens the possibility of using temporal artefacts in LED lamps to influence insect phototaxis without degrading colour rendering.

In the following sections, we describe the setup and results of a field experiment that tested the hypothesis that the flickering of LED light sources at frequencies above the mean CFF value for humans influences the phototaxis of nocturnal flying insects.

\section{Materials and methods}

\subsection{Experimental setup}

The experimental setup consisted of four insect traps placed outdoors at different locations $\left(\mathrm{L}_{1}-\mathrm{L}_{4}\right)$ as indicated in Figure 1(a). Traps at $\mathrm{L}_{1}, \mathrm{~L}_{2}$, and $\mathrm{L}_{3}$ were built using a plastic chamber $(40 \times 25 \times 10 \mathrm{~cm})$ and a lamp separated from the chamber by $20 \mathrm{~cm}$ (Figure 1(b)). Insects were captured in the traps by falling into a solution of water and detergent in the chambers.

The illumination sources used at $\mathrm{L}_{1}$ and $\mathrm{L}_{2}$ were LED lamps (400 lm, $2700 \mathrm{~K}$, dimmable) built at Philips Research to flicker according to the signal injected by a function generator

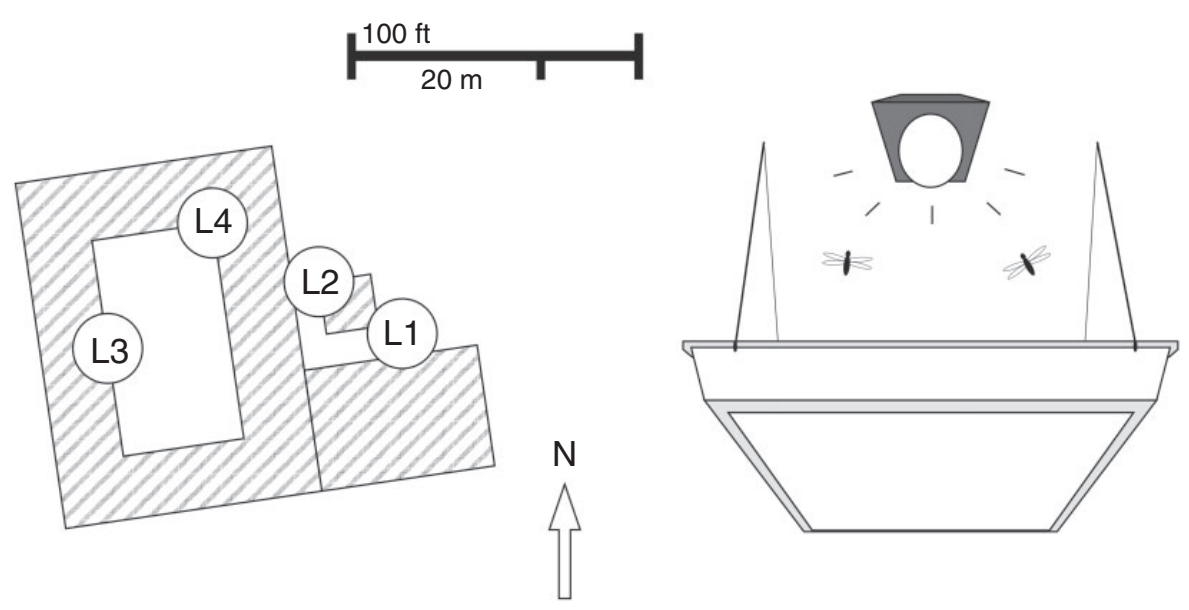

Figure 1 (a) Locations $L_{1}$ and $L_{2}$ hosted the traps with the experimental LED lamps while traps working as positive and negative controls were placed at locations $L_{3}$ and $L_{4}$, respectively. Shaded areas in the figure indicate buildings. (b) An insect trap used in the experiment 
(Agilent Arbitrary Function Generator, Model 33220A, $20 \mathrm{MHz}$ ). For each day of the experiment, one of these lamps was set to flicker according to a square wave, while the other was dimmed to produce an intensitymatching non-flickering output (constant light). Hereafter, we refer to flickering lamps as the experimental intervention group and non-flickering lamp as the control group.

A total of four flickering patterns (experimental conditions) were tested by changing the frequency and duty cycle (DC) of the square wave signal: $120 \mathrm{~Hz} / 10 \%$ DC, $120 \mathrm{~Hz} / 90 \% \quad$ DC,$\quad 240 \mathrm{~Hz} / 10 \% \quad$ DC and $240 \mathrm{~Hz} / 90 \%$ DC.

Each of these four experimental conditions was tested exactly once per week during 15 consecutive weeks, from Monday to Thursday. The order of the conditions tested in a given week was chosen randomly. The result bias caused by a potential systematic difference between the LED lamps or between locations $\mathrm{L}_{1}$ and $\mathrm{L}_{2}$ was minimised by randomly selecting which of the locations, $L_{1}$ or $\mathrm{L}_{2}$, was illuminated by flickering light in a given day.

The trap at $\mathrm{L}_{3}$ was illuminated using a compact fluorescent lamp $(14 \mathrm{~W}, 50-60 \mathrm{~Hz}$, $784 \mathrm{~lm}, 6400 \mathrm{CCT}$, FLC) of fixed setting and was used as a positive control for estimating fluctuations in the population of flying insects attracted to light over time. Conversely, the trap at $\mathrm{L}_{4}$ had a plastic chamber without the lamp and was used as a negative control for assessing whether nocturnal insect capture was influenced by light or merely a result of chance. In order to avoid confusion with the control group of non-flickering LEDs, we will explicitly refer to these traps as positive or negative control when we mention them.

Lamps were lit from dusk to after sunrise. Trap locations were chosen to reduce the illumination interference between traps and with other lamps in the vicinity. Insect collection was carried out on the campus of Universidade Estadual Paulista, Rio Claro,
Brazil, during weekdays from the middle of August (week 33) to the end of November of 2013 (week 47). The experiments did not involve any endangered or protected species.

\subsection{Statistical methods}

We evaluated the statistical evidence of the influence of flickering light on insect phototaxis by performing intraday comparisons of the number of insects captured in different traps. This approach largely removes the confounding effect of weather and other external conditions from the assessment.

In the analysis, we estimated the expected value of the probability $\boldsymbol{\theta}$ of capturing insects in the intervention lamp (as opposed to the control lamp) during an arbitrary day. Given that $\boldsymbol{\theta}$ is random variable representing the intraday probability of capturing insects in the intervention lamp relative to the control lamp, if the bias $E(\boldsymbol{\theta})$ is larger than $50 \%$, then the intervention condition attracts on average more insects than the control lamp. The converse is true if $E(\boldsymbol{\theta})$ is lower than $50 \%$.

We used a hierarchical Bayesian model (see Appendix) in order to calculate the probability of $E(\boldsymbol{\theta})$ being larger (or lower) than $50 \%$. The model estimates the distribution of $E(\boldsymbol{\theta})$ conditioned to the total number of insects captured in control and intervention lamps per day of collection and the number of insects found at the intervention lamp per day of collection.

The probability distribution of the bias conditioned to the data observed was computed via Markov Chain Monte Carlo simulations using JAGS 3.3.0 and R 2.15.0.

\section{Results}

The number of insects captured at $\mathrm{L}_{1}$ and $\mathrm{L}_{2}$ was 12,320 and its time distribution followed a pattern that reflects the seasonality of insect population in the region. As seen in Figure 2, the number of insects captured in $\mathrm{L}_{1}$ and $\mathrm{L}_{2}$ 


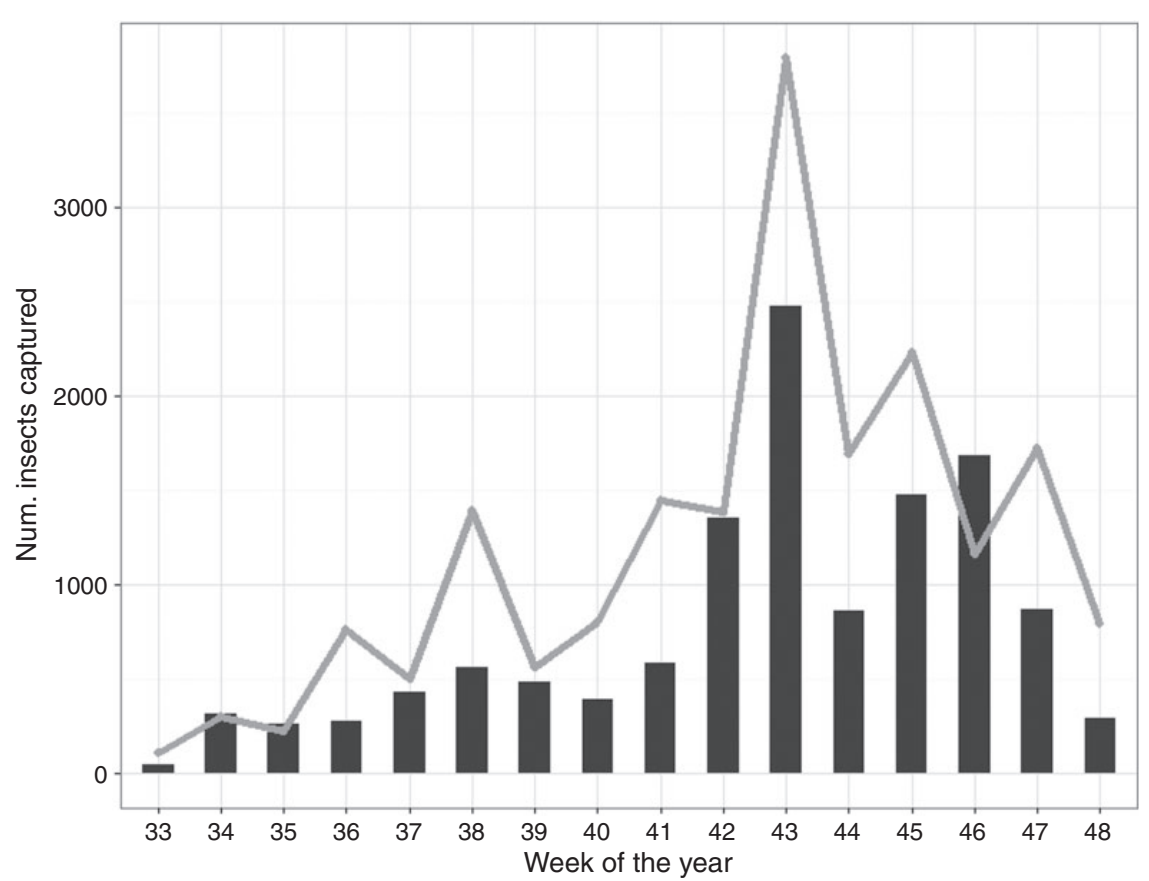

Figure 2 Total number of insects captured per week at $\mathrm{L}_{1}+\mathrm{L}_{2}$ (bars represent the sum of specimens in these traps) and at the positive control location $\mathrm{L}_{3}$ (line)

(black bars) and in the positive control $\mathrm{L}_{3}$ (grey line) slowly increases towards the end of winter (week 38) before accelerating in spring (weeks 40 to 43). Figure 2 also indicates that $\mathrm{L}_{3}$ consistently outperformed $\mathrm{L}_{1}$ and $\mathrm{L}_{2}$ in the number of specimens captured. This result is consistent with previous observations that the spectrum of CFL lamps attract more insects than does that of LEDs and that attraction increases with light intensity. ${ }^{7,8,13}$ In the discussion that follows, we only consider specimens captured at $\mathrm{L}_{1}$ and $\mathrm{L}_{2}$ since the lamps at these traps differ exclusively by the presence or absence of flickering.

The insect orders with highest numbers of captured specimens were, respectively, Diptera $(n=5809)$, Hemiptera $(n=2073)$, Hymenoptera $\quad(n=1527), \quad$ Coleoptera $(n=922)$ and Isoptera $(n=882)$. The distribution of insect orders over time is depicted in Figure 3.
A comparison of the total number of insects captured per experimental condition indicated that the overall number of insects caught in traps with flickering lighting was lower than in traps with constant light output, independent of the combination of DC and flicker frequency tested. In the following discussion, we use the symbol $\Delta$ to denote the relative difference between the total number of insects captured in the control and intervention conditions.

The largest relative difference between control and intervention conditions, $\Delta=-33.6 \%$, was observed for $120 \mathrm{~Hz}$ and $10 \%$ DC and the lowest, $\Delta=-12.9 \%$, for $240 \mathrm{~Hz}$ and $90 \%$ DC. The differences observed for conditions $120 \mathrm{~Hz} / 90 \%$ DC and $240 \mathrm{~Hz} / 10 \%$ DC were, respectively, $\Delta=-24.4 \%$ and $\Delta=-23.2 \%$.

The relative difference (decrease or increase) of the total number of insects 


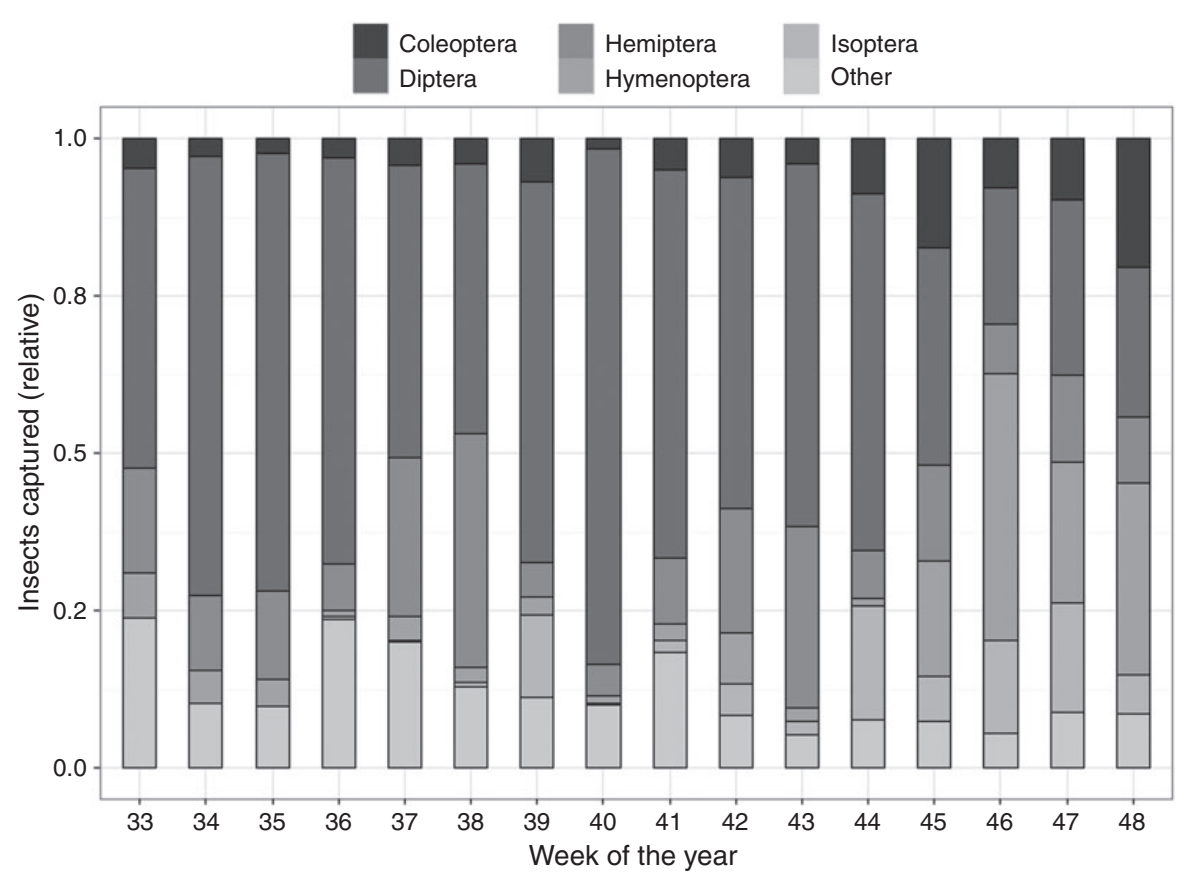

Figure 3 Composition of insects per week for most captured insect orders at $L_{1}$ and $L_{2}$ (all orders that contribute less than $5 \%$ of total capture are grouped as 'Other')

captured in the experimental conditions in comparison to control is shown in Figure 4 discriminated by insect order. The numbers indicate large variations in the magnitude and direction of the effects observed. A consistent decrease of specimens captured across flickering conditions was observed in Diptera, Coleoptera and Psocoptera. The remaining orders Hemiptera, Hymenoptera, Isoptera and Lepidopera displayed a decrease or an increase of insect capture depending on the flickering adopted.

The relative comparison of the number of insects captured in the experimental conditions and in the negative control located at $\mathrm{L}_{4}$ is shown in Figure 5. Independent of the insect order observed or flicker setting used, more insects were captured in the illuminated traps. We thus found no evidence that the flickering conditions tested are able to exert a repelling effect with respect to darkness.

\section{Statistical analysis}

The comparison of lamps using the accumulated number of specimens captured over multiple days, as presented in the previous graphs, is very sensitive to atypical events: a single anomalous day may alter the direction of the comparison and mask the underlying systematic differences between the tested conditions. In order to uncover the systematic difference between control and intervention conditions, we estimated the intraday expected probability of capturing insects in the intervention lamp (as opposed to the control lamp) using a hierarchical Bayesian model (see Appendix).

Figure 6 summarises the results of the analysis by displaying the expected relative difference (increase or decrease) of the number of insects captured in the intervention condition in comparison to the control 


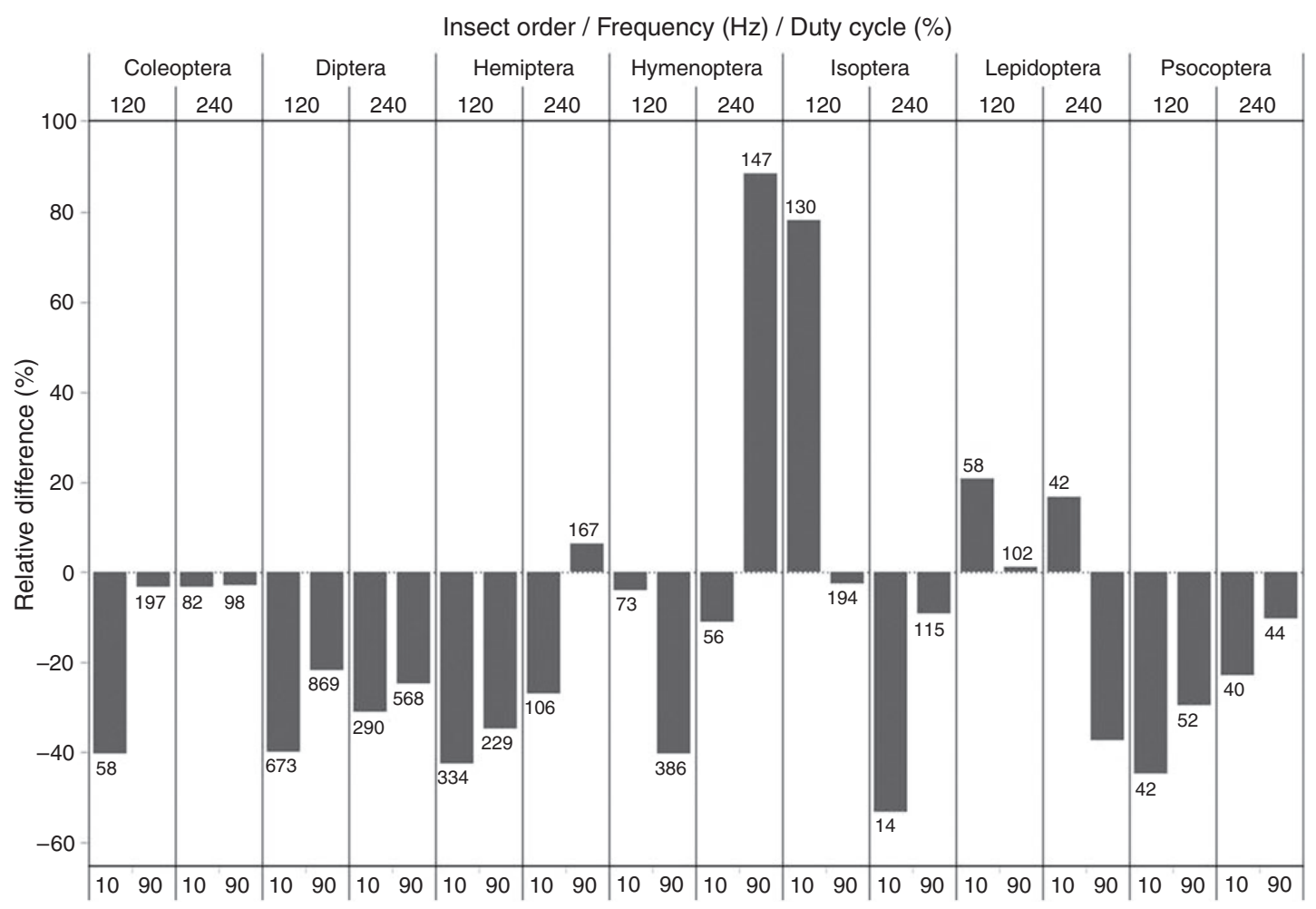

Figure 4 Relative difference (decrease or increase) $\Delta$ in the total number of insects captured in the experimental condition in comparison to the control (non-flicker) condition. The values on the bars show the total number captured in the experimental condition

condition. Also indicated are the probabilities that the estimated values are different from zero and in the right direction, i.e. positive when the flickering condition indeed captures more insects, and negative otherwise. We consider a probability larger than $95 \%$ as statistically significant. According to this criterion, a significant reduction of attraction by flickering was observed for Diptera $(120 \mathrm{~Hz} / 10 \%$ DC), Hemiptera $(120 \mathrm{~Hz} / 90 \%$ DC) and Lepidopera ( $240 \mathrm{~Hz} / 90 \%$ DC).

For specific light flickering conditions, the overall numbers of Hemiptera, Psocoptera, Hymenoptera and Isoptera specimens captured in traps with flickering light were higher than those in traps with non-flickering light, although such differences were not statistically significant according to our analysis.

\section{Discussion}

A one to one comparison between the aggregated results over multiple days (Figure 4) and the intraday expected effects of light flickering (Figure 6) indicates overall agreement in the direction of the effect (positive or negative). Where the tables disagree, e.g. Lepidoptera $120 \mathrm{~Hz} / 90 \%$ DC, the probability derived from the Bayesian model for the direction of the effect was low (between 50-65\%).

At the onset of the experiment, we expected to observe a lower $\Delta$ for traps illuminated with flickering light at $240 \mathrm{~Hz}$ than those at $120 \mathrm{~Hz}$ since higher frequencies are less likely to be below the insect CFF, i.e. perceived as a train of pulses. This expectation was 


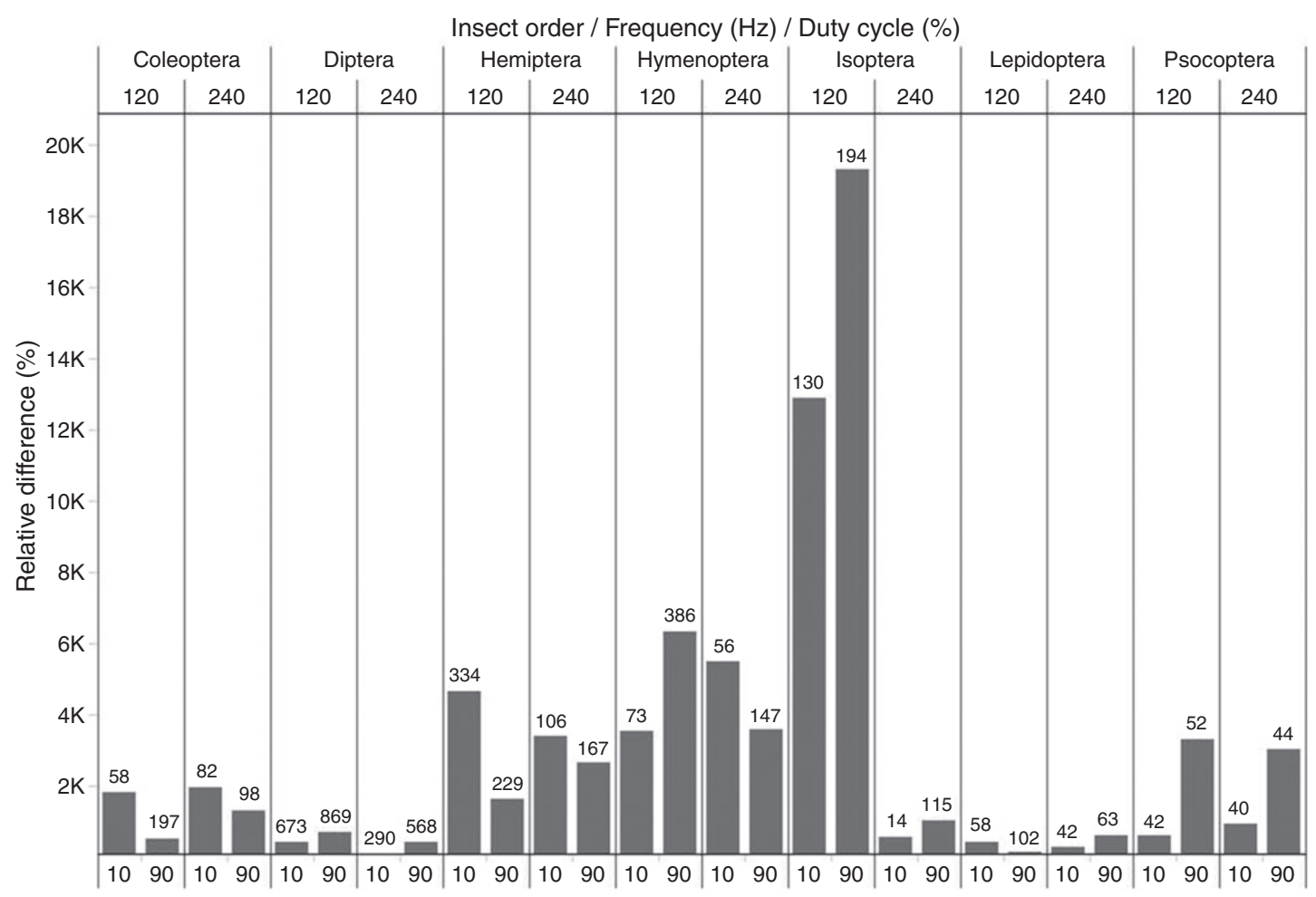

Figure 5 Relative increase $\Delta$ in the total number of insects captured in the experimental condition in comparison to the negative control condition at $\mathrm{L}_{4}$. The values on the bars show the total number captured in the experimental condition

confirmed when we compared frequencies both at $10 \% \quad \mathrm{DC}, \quad \Delta=-33.6 \% \quad(120 \mathrm{~Hz})$ versus $\Delta=-23.2 \% \quad(240 \mathrm{~Hz})$, and at $90 \% \mathrm{DC}, \Delta=-24.4 \%(120 \mathrm{~Hz})$ versus $\Delta=$ $-12.9 \%(240 \mathrm{~Hz})$.

The Illuminating Engineering Society of North America provides recommendation on the acceptable amount of flicker for humans based on the Flicker Index (FI), defined as the area above the average light output divided by its total area for a single cycle of the fluctuation. ${ }^{14}$ The FI may vary between 0 and 1 with recommended values equal to 0.1 or less. In our experimental setup, flicker with DC of $10 \%$ and $90 \%$ corresponds to a FI of 0.9 and 0.1 , respectively, suggesting a higher flicker nuisance for the former. Although no recommended values for FI exist for insects, our results suggest that a higher FI is more effective in deterring insect attraction. We observed higher $\Delta$ value for traps illuminated at $10 \%$ DC than for those at $90 \%$, both for $120 \mathrm{~Hz}, \quad \Delta=-33.6 \% \quad(10 \% \quad$ DC $)$ versus $\Delta=-24.4 \% \quad(90 \%$ DC), and for $240 \mathrm{~Hz}$, $\Delta=-23.2 \%(10 \%$ DC) versus $\Delta=-12.9 \%$ $(90 \%$ DC). For Hymenoptera, however, it was flicker at a $90 \%$ DC that seemed more effective in affecting insect attraction (cf. Figures 4 and 6).

The effect of flickering light on insect phototaxis has been tested by several studies under experimental conditions, mostly aiming to attract pest insects to light sources in order to develop environmentally friendly traps for houseflies. ${ }^{15-17}$ Although enhanced attraction to flickering light has been reported for 


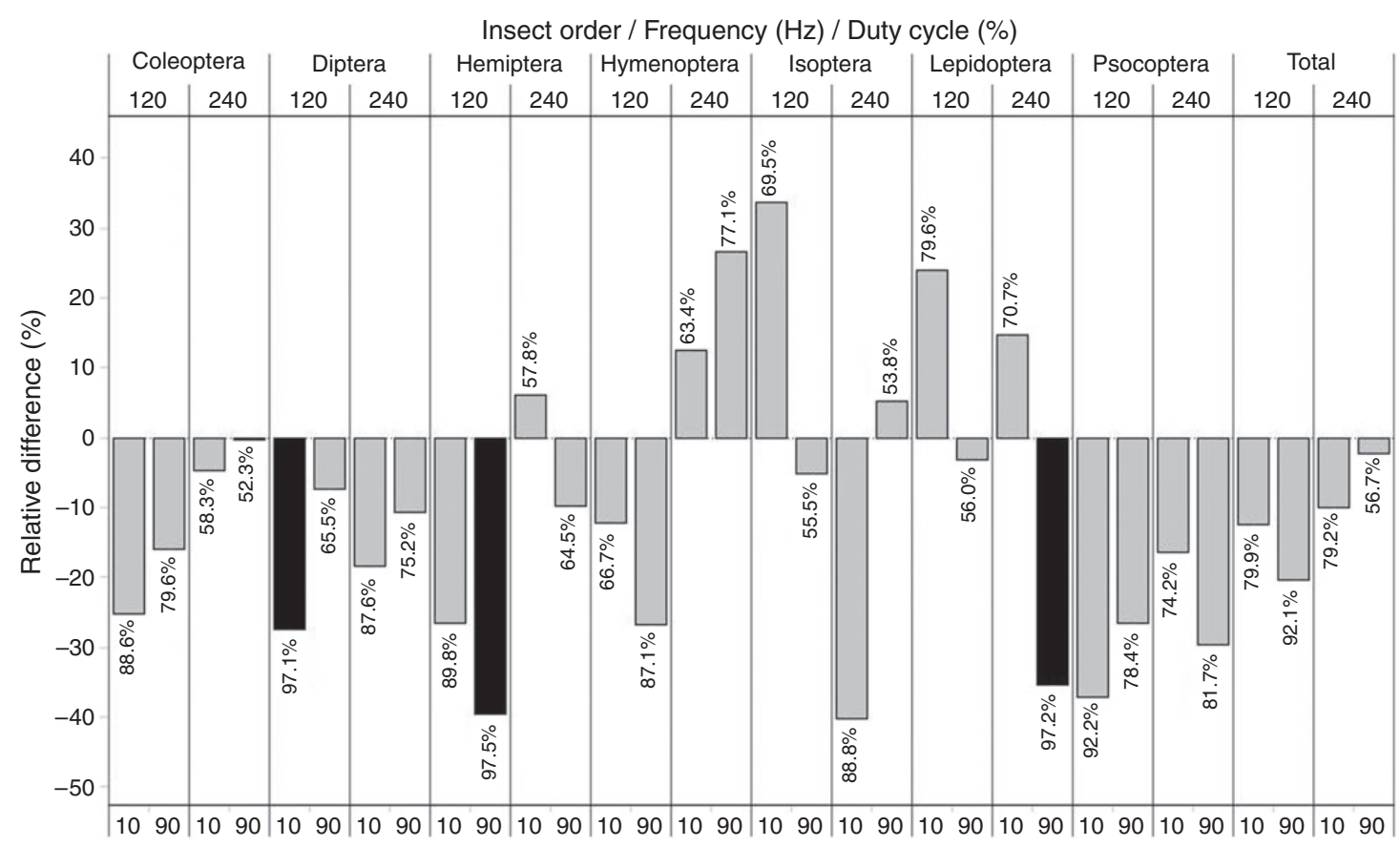

Figure 6 Expected difference (increase or decrease) caused by flicker on the number of insects captured as a percentage of the control (non-flicker) condition. The number at the end of each bar indicates the probability that the expected value is in the direction depicted - positive or negative. Black bars indicate statistically significant differences

houseflies under low-depth modulated light, the deep modulation used in our study is likely to introduce stroboscopic effects that impair visual orientation during movement. Stroboscopic effect refers to the interaction of moving objects with temporally modulated light and may explain why honeybees display a reduction of flight and orientation abilities under flickering light. ${ }^{11,18}$

Although the survey of Inger et al. ${ }^{12}$ reports a mean CFF value for nocturnal insects quite close to that of humans, thus portraying nocturnal insects as largely insensitive to flicker invisible to humans, this value represents an average of a very heterogeneous group. Nocturnal flying insects, for example, are likely to display higher CFF values than those of slow moving species since the former require faster visual temporal resolution to manoeuvre during flight. In addition, many species which are active during the night initiate their activity around dusk when there is still plenty of light available. These species are likely to display higher CFF values than purely nocturnal species. They are also likely to be the group most affected by artificial lighting, as the natural behaviour and orientation of duskactive species seem to depend on subtle changes in natural light levels. We thus reasonably expect that the species most affected by artificial light and most capable of quickly moving towards light sources are precisely those with the highest CFF values.

The potential applications of the results of this study to influence the behaviour of insects must be balanced against previous findings on the effects of flicker on humans and other animals. ${ }^{12}$ For humans, these effects may include epileptic seizures for 
frequencies below $100 \mathrm{~Hz}$ as well as headaches and impaired visual performance for frequencies up to the $\mathrm{kHz}$ range. Flicker of about or above $100 \mathrm{~Hz}$ has also been suggested to be perceived by squirrels, pigeons and domestic chickens with unknown consequences for the species affected. Also very little is known about CFFs of wild birds as very few species have been tested.

Commercial light sources usually flicker, albeit at imperceptible frequencies. Incandescent lamps driven by an alternate current at $50-60 \mathrm{~Hz}$ will flicker at double the mains frequency or $100-120 \mathrm{~Hz}$. Fluorescent lamps with older magnetic ballast also flicker at the same frequency, but the advent of electronic ballasts enabled them to flicker at much higher frequencies, e.g. 30,000 Hz. Both incandescent and fluorescent lamps usually display flickering with low modulation depth, i.e. ratio between peak value and the difference between peak and minimum value, due to the physics of light generation. Commercial LED lamps at full intensity or dimmed via analogue methods have a modulation depth that depends on the characteristics of their driver. LEDs equipped with low-end drivers, e.g. alternating current light emitting diodes (ACLEDs), operate directly using AC mains power and small resistors instead of expensive circuitry. Although such LEDs are more efficient as no power is lost in the $\mathrm{AC}$ to DC conversion, their light output typically fluctuates at twice the supply frequency (usually between 100 and $120 \mathrm{~Hz}$ ). ACLED light shows deep modulation because of their fast response to changing current. High-end drivers include additional electronic components to enable very low modulation depth and the elimination of temporal artefacts. ${ }^{19}$ As with ACLEDs equipped with low-end drivers, the flicker in our experiment was deeply $(100 \%)$ modulated.

Besides temporal artefacts derived from the choice of AC to DC conversion, many LEDs are equipped with circuitry to control brightness by imposing an on/off pattern to the light output at a frequency that is not perceived by humans, a process known as pulse wave modulation. As pulse wave modulation circuitry of LED lamps can be configured to produce flicker at specific frequencies and DCs, tuning flicker to influence insect phototaxis can be done without the need for extra electronics.

\section{Conclusions}

The results of the experiment show that fewer insects were captured in the trap illuminated with the flickering light source, independent of the flicker setting used. A statistical analysis of the daily capture indicates an expected significant reduction (at 95\% confidence level) of the number of insects captured in flickering lamps for different combinations of flicker setting and insect order. In particular, a significant reduction of capture is expected in Diptera for $120 \mathrm{~Hz}, 10 \%$ DC; Hemiptera for $120 \mathrm{~Hz}, 90 \%$ DC and Lepidoptera for $240 \mathrm{~Hz}, 90 \%$ DC. The expected reduction in capture for these settings and orders was in the range from $27.4 \%$ to $39.6 \%$. No evidence of positive phototaxis reversal was observed through flickering, i.e. insects being repelled.

The mechanism of action of light flickering on the phototaxis of flying nocturnal insects is not known, but it is hypothesised to be at least in part related to stroboscopic perceptual effects on the flight control of these insects. Since stroboscopic effects will depend on the flicker fusion frequency and flight speed of each insect, this hypothesis can explain why different orders may respond differently to a same flickering pattern.

The results obtained suggest that light flickering can be used to reduce the number of nocturnal insects attracted to the human habitat thus mitigating the impact of artificial light on the surrounding ecosystems and potentially reducing the spread of diseases transmitted via insect vectors. Future work 
will address the effect of flickering on the phototaxis of specific groups of insects, e.g. disease vectors.

\section{Funding}

The authors thank Royal Philips Electronics and FUNDUNESP $(107 / 2013$ - CCP/PIB) for financial support.

\section{References}

1 Barghini A, de Medeiros BAS. Artificial lighting as a vector attractant and cause of disease diffusion. Environmental Health Perspectives 2010; 118: 1503-1506.

2 Pacheco-Tucuch FS, Ramirez-Sierra MJ, Gourbière S, Dumonteil E. Public street lights increase house infestation by the Chagas disease vector Triatoma dimidiata. PLoS One 2012; 7 : e36207.

3 Eisenbeis G, Eick K. Attraction of nocturnal insects to street lights with special regard to LEDs: Society for Conservation Biology, 24th Annual Meeting 2010, University of Alberta in Edmonton, Canada, July 3-7, 2010. Retrieved 10 April 2011, from http://www. conbio. org/ activities/meetings/2010/images/abstracts. pdf.

4 Menzes R, Greggers U. Natural phototaxis and its relationship to colour vision in honeybees. Journal of Comparative Physiology A 1985; 157: 311-321.

5 Cleve K. Der Anflug der Schmetterlinge an künstliche lichtquellen. Mitteilungen der Deutsche Entomologischen Gesellschaft 1964; 23: 66-76.

6 Longcore T, Aldern H, Eggers J, Flores S, Franco L, Hirshfield-Yamanishi E, Petrinec L, Yan W, Barroso A. Tuning the white light spectrum of light emitting diode lamps to reduce attraction of nocturnal arthropods.

Philosophical Transactions of the Royal Society of London B: Biological Sciences 2015; 370: 20140125.

7 Poiani S, Dietrich C, Barroso A, CostaLeonardo AM. Effects of residential energysaving lamps on the attraction of nocturnal insects. Lighting Research and Technology 2015; 47: 338-348.

8 van Langevelde F, Ettema JA, Donners M, WallisDeVries M, Groenendijk D. Effect of spectral composition of artificial light on the attraction of moths. Biological Conservation 2011; 144: 2274-2281.

9 Barghini A, de Medeiros BAS. UV radiation as an attractor for insects. Leukos 2012; 9: 47-56.

10 Huemer P, Kühtreibe H, Tarmann G. Anlockwirkung moderner Leuchtmittel auf nachtaktive Insekten: Ergebnisse einer Feldstudie in Tirol. Innsbruck: Tiroler Landesmuseum, 2010.

11 Frantsevich LI, Mokrushov PA. Orientation of bees with stroboscopic presentation of figures. Doklady Akademii nauk SSSR 1970; 194: 734-736.

12 Inger R, Bennie J, Davies T, Gaston K. Potential biological and ecological effects of flickering artificial light. PloS One 2014; 9: e98631.

13 Ferreira MT, Scheffrahn RH. Light attraction and subsequent colonization behaviors of alates and dealates of the West Indian drywood termite (Isoptera: Kalotermitidae). Florida Entomologist 2011; 94: 131-136.

14 Illuminating Engineering Society of North America. Lighting Handbook: Reference and Application. 9th Edition, New York: IESNA, 2000.

15 Syms PR, Goodman LJ. The effect of flickering U-V light output on the attractiveness of an insect electrocutor trap to the house-fly, Musca domestica. Entomologia Experimentalis et Applicate 1987; 43: 81-85.

16 Chu C-C, Chen T-Y, Henneberry TJ. Attractiveness of flickering and non-flickering cool white fluorescent light to Culex quinquefasciatus (Diptera: Culicidae), Musca domestica (Diptera: Muscidae) and Pectinophora gossypiella (Lepidoptera: Gelechiidae) adults, and Acheta domesticus (Orthoptera: Gryllidae) and Periplaneta americana (Blattodea:

Blattidae) nymphs. Southwestern Entomologist 2006; 31: 77-81.

17 Smallegange RC. Attractiveness of Different Light Wavelengths, Flicker Frequencies and Odours to the Housefly (Musca domestica L.). Groningen, the Netherlands: Rijksuniversiteit Groningen, 2003. 
18 Nilsson DE. The physiology of the compound eyes of insects and crustaceans: by Sigmund Exner. Trends in Neurosciences 1990; 13: 470-471.

19 Perz M, Vogels IMLC, Sekulovski D, Wang L, Tu Y, Heynderickx IEJ. Modeling the visibility of the stroboscopic effect occurring in temporally modulated light systems. Lighting Research and Technology 2015; 47: 281-300.

\section{Appendix: Hierarchical Bayesian model}

Let $\boldsymbol{\theta}_{i}$ be the probability of capturing insects in the intervention lamp (as opposed to the control lamp) within an arbitrary day $i$. In the analysis of the effects of flickering on insect capture, we assess systematic differences between the control and intervention conditions by estimating the expected value of $\boldsymbol{\theta}_{i}$, henceforth represented as $\mu=E\left(\boldsymbol{\theta}_{i}\right)$. We use a hierarchical Bayesian model to estimate the conditional distribution of $\mu$ given observed values $n_{i}$ and $N_{i}$ for each day of the experiment, where $N_{i}$ represents the total number of insects captured in both control and intervention lamps and $n_{i}$ is the number of insects found at the intervention lamp only.

Given $\boldsymbol{\theta}_{i}$ and assuming insects are captured independently from each other, the number of insects $n_{i}$ found at the intervention lamp during day $i$ is a random variable with binomial distribution, i.e. $n_{i} \sim \operatorname{binomial}\left(N_{i}\right.$, $\left.\boldsymbol{\theta}_{i}\right)$. Furthermore, as external conditions such as wind direction, moonlight, etc., may change from day to day, probability $\boldsymbol{\theta}_{i}$ is a random variable modelled by a beta distribution, i.e. $\boldsymbol{\theta}_{i} \sim \operatorname{beta}(a, b)$. The mean of this beta distribution is by definition $\mu$ or the systematic bias in insect attraction created by the intervention. Parameters $a$ and $b$ can be written as $a=\mu K$ and $b=(1-\mu) K$ where $K$ is a factor inversely proportional to the standard deviation.

In the Bayesian model, $\mu$ and $K$ are themselves random variables whose priors are respectively a beta and a gamma distribution, i.e. $\mu \sim \operatorname{beta}\left(A_{\mu}, \quad B_{\mu}\right)$ and $K \sim \operatorname{gamma}\left(S_{K}, R_{K}\right)$. We set these priors to reflect previous research that observed little impact of flickering on the phototaxis of flies ${ }^{15}$ by choosing $A_{\mu}=2 ; B_{\mu}=2$ and $S_{K}=1$; $R_{K}=0.1$.

The hierarchical model is thus specified by the following random variables and dependencies:

$$
\begin{aligned}
\theta_{i} & \sim \operatorname{beta}(a, b), i=1,2, \ldots \\
a_{i} & =\mu K ; b=(1-\mu) K \\
\mu & \sim \operatorname{beta}\left(A_{\mu}, B_{\mu}\right) ; K \sim \operatorname{gamma}\left(S_{K}, R_{K}\right) \\
\mathrm{A}_{\mu} & =2 ; B_{\mu}=2 ; S_{K}=1 ; R_{K}=0.1
\end{aligned}
$$

The probability distribution of $\mu$ conditioned to data observed was computed via Markov Chain Monte Carlo simulation. A 5000 burn-in period was used to dilute the influence of initial values on the results. From the distribution of $\mu$, its expected value and probability that $\mu$ is greater (or lower) than 0.5 are computed. The expected value of $\mu$ offers an estimation of the systematic difference between control and intervention conditions, while the probability that $\mu$ is greater (or lower) than 0.5 indicates the confidence on the difference. 